\title{
Ultrafast low-energy all-optical switching using a photonic-crystal asymmetric Fano structure
}

\author{
Yu, Yi; Hu, Hao; Oxenløwe, Leif Katsuo; Yvind, Kresten; Mørk, Jesper
}

Published in:

Proceedings of 2015 International Conference on Photonics in Switching

Link to article, DOI:

10.1109/PS.2015.7328964

Publication date:

2015

Document Version

Peer reviewed version

Link back to DTU Orbit

Citation (APA):

Yu, Y., Hu, H., Oxenløwe, L. K., Yvind, K., \& Mørk, J. (2015). Ultrafast low-energy all-optical switching using a photonic-crystal asymmetric Fano structure. In Proceedings of 2015 International Conference on Photonics in Switching (pp. 94-96). IEEE. https://doi.org/10.1109/PS.2015.7328964

\section{General rights}

Copyright and moral rights for the publications made accessible in the public portal are retained by the authors and/or other copyright owners and it is a condition of accessing publications that users recognise and abide by the legal requirements associated with these rights.

- Users may download and print one copy of any publication from the public portal for the purpose of private study or research.

- You may not further distribute the material or use it for any profit-making activity or commercial gain

- You may freely distribute the URL identifying the publication in the public portal 


\title{
Ultrafast low-energy all-optical switching using a photonic-crystal asymmetric Fano structure
}

\author{
Yi Yu, Hao Hu, Leif Katsuo Oxenløwe, Kresten Yvind, and Jesper Mork \\ DTU Fotonik, Department of Photonics Engineering \\ Technical University of Denmark DK-2800 \\ Kongens Lyngby, Denmark \\ yiyu@fotonik.dtu.dk
}

\begin{abstract}
We experimentally demonstrate $20 \mathrm{Gbit} / \mathrm{s}$ alloptical switching with low-energy consumption using a simple and ultra-compact InP photonic-crystal structure by employing a well-engineered Fano resonance in combination with broken mirror symmetry.
\end{abstract}

Keywords-Nonlinear optical devices, Photonic crystals, Alloptical devices

\section{INTRODUCTION}

Ultra-compact photonic structures that perform optical signal processing such as modulation and switching at highspeed with low-energy consumption are essential for enabling integrated photonic chips that can meet the growing demand for information capacity [1], [2]. In cavity-based switches, an applied control signal changes the refractive index of the cavity, thereby shifting the cavity resonance and modulating the transmission of the data signal [3]. The shape of the transmission spectrum is important, since it determines the refractive index shift, and thereby the control energy, required for achieving a certain on-to-off ratio [3]-[5].

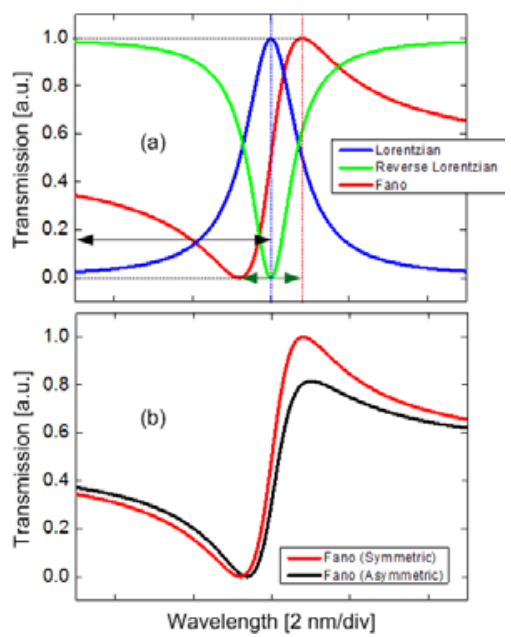

Fig. 1. (a) Comparison of transmission spectra for structures with Lorentzian and Fano-type resonance. The $Q$-factors and resonance wavelengths are the same. (b) Transmission spectra for Fano resonance with and without mirror symmetry.

As shown in Fig. 1(a), the extended tails of a Lorentzian spectrum imply large switching energies, since the resonance needs to be shifted significantly to properly switch the signal. In contrast, a Fano resonance [6], [7] has an asymmetric spectrum, featuring a large transmission change within a narrow wavelength range, determined by the transition from constructive to destructive interference between the discrete resonance and the continuum, thus enabling low-energy switching. The advantage of a Fano resonance was recently demonstrated using a symmetric photonic-crystal (PhC) structure employing an H1-type cavity [8]. It was also found that the non-monotonous frequency-dependence of the Fano transmission spectrum implies an inherent reduction of patterning effects. In contrast, a Lorentzian spectrum, with its monotonously-varying tails, converts (slow) dynamics of the resonance shift into amplitude modulation, limiting the bitrate due to the long carrier lifetime [9]-[14].
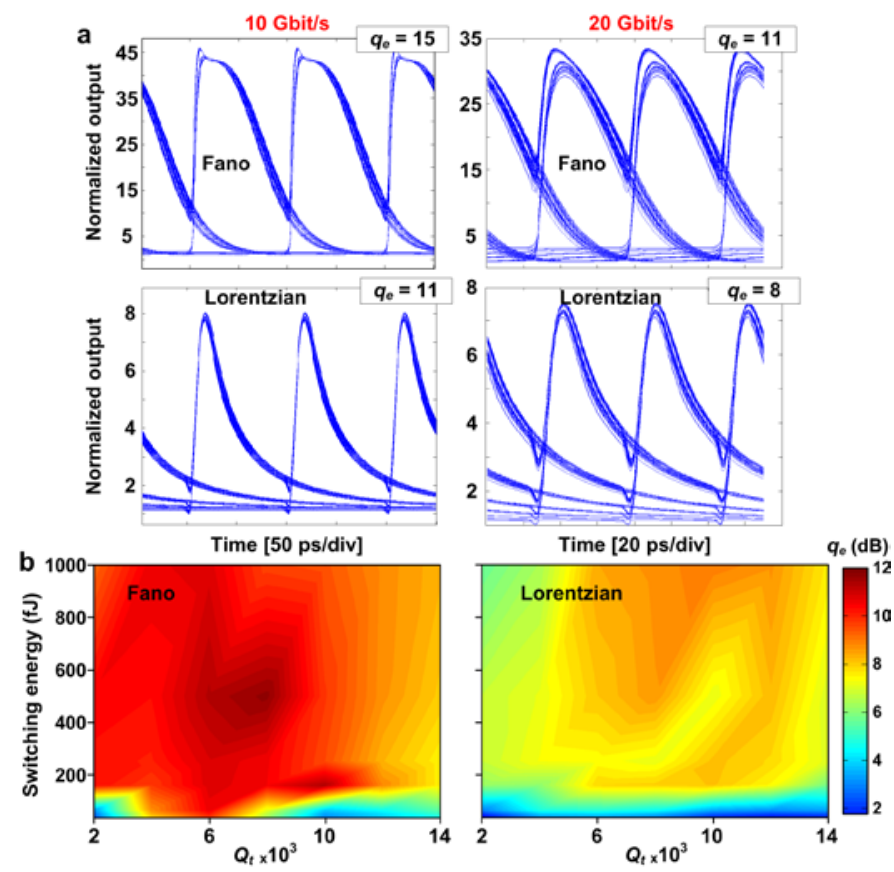

Fig. 2. (a) Calculated eye diagrams of the converted signal for comparable Lorentzian and Fano structure with the same $Q$-factor at 10 and $20 \mathrm{Gbit} / \mathrm{s}$. The other conditions are the same. The extracted values of the quality factor $q_{e}$ (in $\mathrm{dB}$ ) of the eye diagram are given in the figures. (b) Contour plots of the largest achievable value of $q_{e}$ at $40 \mathrm{Gbit} / \mathrm{s}$ upon variation of total $Q$-factor $Q_{t}$ and pump energy, for the Fano (left) and Lorentzian (right) structure. The intrinsic $Q$-factor of the two structures is set infinite.

Fig. 2 numerically compares the high-speed performance of a comparable Fano and Lorentzian structure for aperiodic 
pump signals. It is seen that the Fano structure has a considerably larger eye opening than the Lorentzian structure, which is ascribed to the suppression of the slow decay component. We quantify the performance by calculating a quality factor $q_{e}$ from the eye diagram, a measure of the distortion of the eye due to patterning effects. The Fano structure is seen to result in a larger eye opening than the Lorentzian over a wide range of cavity $Q$-factors.

\section{DEVICE DESGIN}

Here we experimentally demonstrate that by breaking the mirror symmetry of a Fano structure, the switching properties can be further improved. Fig. 3(a) shows the fabricated $\mathrm{PhC}$ InP membrane with a thickness of $340 \mathrm{~nm}$, lattice constant of $447 \mathrm{~nm}$ and hole radius $R=113 \mathrm{~nm}$. An H0-type cavity is sidecoupled to a standard W1-defect type waveguide. The device is equipped with mode adapters to facilitate out coupling [15]. The detailed fabrication processes were described in Ref. [16]. The structure has two key features. First, one blockade hole $(\mathrm{BH})$, acting as a partially transmitting element (PTE), is added in the waveguide above the nanocavity, with a transmission coefficient $t_{B}^{2}$ that can be varied via the radius $R_{B}$ (here $R_{B}=1.2 R$ ) of the $\mathrm{BH}$ [17], [18]. This makes the structure exhibit an asymmetric Fano line. Fig. 3(b) shows the spectrum, calculated using a three-dimensional finitedifference time-domain (FDTD) technique, exhibiting an onoff contrast of more than $30 \mathrm{~dB}$ within a narrow wavelength range. Second, the mirror symmetry of the structure is broken by displacing the $\mathrm{BH}$ by one lattice constant towards port 1 . This makes the coupling rate $\gamma_{1}$ between port 1 and cavity, differ from the rate $\gamma_{2}$ between port 2 and cavity. Recently, a similar structure with broken symmetry was shown to enable non-reciprocal light transmission when combined with an optical nonlinearity [19]. For the present design, FDTD simulations lead to $t_{B}=0.24$ and $\gamma_{1} / \gamma_{2}=2.5$. Transmission from port 1 (2) to port 2 (1) is henceforth denotes as forward (backward).

We first analyze the dynamical properties of symmetric and asymmetric Fano structures using nonlinear temporal coupled mode theory [17]-[20]. We compare the results for a symmetric $\left(\gamma_{1} / \gamma_{2}=1\right)$ and an asymmetric $\left(\gamma_{1} / \gamma_{2}=2.5\right)$ Fano structure with the same unloaded $Q$-factor $Q_{v}$ of $1.2 \times 10^{5}$ and total $Q$-factor $Q_{t}$ of $1.0 \times 10^{3}$, cf. Fig. 4 . These two structures give almost identical Fano transmission spectra. A weak continuous wave (CW) signal is applied to the structure together with a $10 \mathrm{GHz}$ train of pump pulses (12 ps) and energies of $57 \mathrm{fJ}$. Fig. 4 shows the simulated cavity energy and output signal power as a function of time. Compared to the symmetric Fano structure, larger cavity energy and switching contrast are achieved when operating the asymmetric Fano structure in the forward direction, i.e. injecting from port 1. This is due to the switching improvements that can be obtained using asymmetric structures where larger energy can be coupled into the cavity, without scarificing the $Q$-factor. Simulations for other operation conditions show that the advantage of asymmetric structures is a common feature. This result seems counterintuitive since the asymmetry of the coupling coefficients can result into a lower transmission, cf. Fig. 1(b). However, the improvement in the nonlinear switching performance compensates for this additional transmission loss.

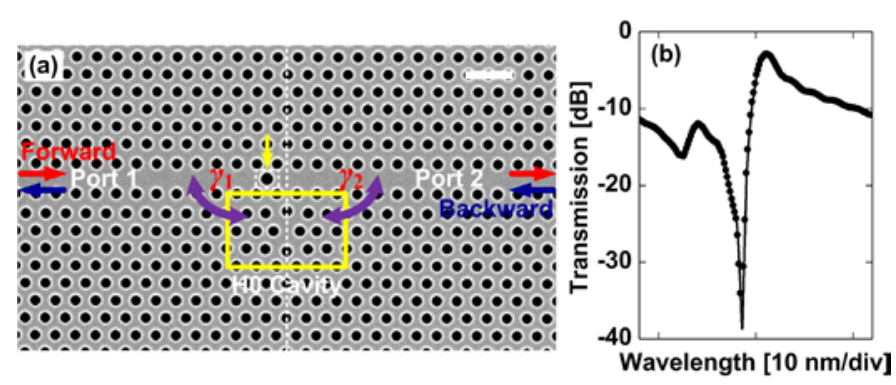

Fig. 3. (a) Fabricated InP membrane structure. The scale bar corresponds to $1 \mu \mathrm{m}$. The structure is made asymmetric with respect to the mid-plane (vertical dashed line) by displacing the $\mathrm{BH}$ one lattice constant towards port 1. (b) Example of FDTD-simulated transmission spectrum of the structure showing a Fano resonance.

\section{EXPERIMENTAL RESULTS}

Next, we experimentally investigate the all-optical switching properties of the structure at 10 and $20 \mathrm{Gbit} / \mathrm{s}$. RZ pump pulses (10 ps) are first generated and then modulated in the OOK format at 10 and $20 \mathrm{Gbit} / \mathrm{s}$ with a PRBS with the length of $2^{31}-1$. The modulated pump and a CW signal are coupled into the $\mathrm{PhC}$ device through port 1 . At the output of the device, i.e. port 2 , the combined signal is amplified before the modulated signal is separated from the pump using an optical band-pass filter, and subsequently detected by a receiver. The pump and signal are slightly red-detuned from the Fano resonance maximum and minimum, respectively. Fig. 5(a) shows the measured BER curves for $10 \mathrm{Gbit} / \mathrm{s}$ alloptical modulation. The BER decreases as the pump energy increases due to an enhanced switching contrast. Error-free $\left(\mathrm{BER}<10^{-9}\right)$ operation is achieved with a coupled pump energy of only $60 \mathrm{fJ} / \mathrm{bit}$, which is several times lower than the result obtained using an H1-type cavity with a symmetric configuration [8]. This low energy consumption is mainly ascribed to the asymmetric configuration of the device. Besides, a larger $Q / V$ value of the $\mathrm{H} 0$ cavity also acts to reduce the switching energy.

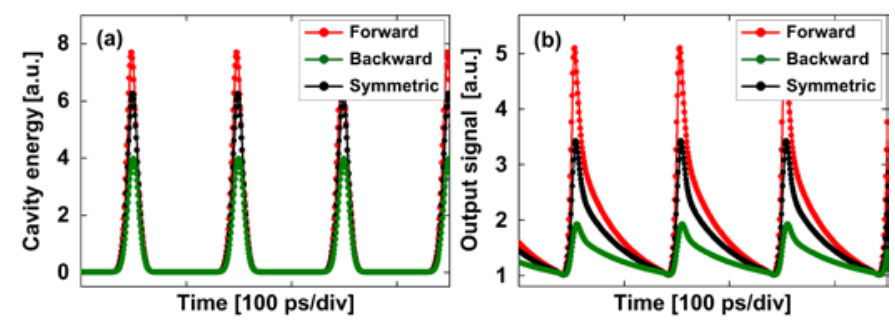

Fig. 4. Simulated (a) cavity energy and (b) output signal power versus time for a $10 \mathrm{GHz}$ pump pulse train (normalized to 1 at their minimum values). The red (green) dotted lines show the forward (backward) transmission for an asymmetric Fano structure, and the black dotted lines show the results for a symmetric Fano structure. The input signal is $0.4 \mathrm{~nm}$ red detuned from the transmission minimum of the Fano spectrum, while he pump pulses, with a Gaussian envelope, are tuned to the cavity resonance.

In Fig. 5(a), a power penalty of $\sim 3 \mathrm{~dB}$ is seen at $10^{-9} \mathrm{BER}$ for a pump energy of $151 \mathrm{fJ} / \mathrm{bit}$. For $20 \mathrm{Gbit} / \mathrm{s}$ operation, cf. Fig. 5(b), the BER increases somewhat, but is still well below 
the threshold for employing forward error correction (FEC) schemes. Compared with a traditional Lorentzian structure [11], we find that patterning effects are significantly suppressed and the energy consumption is reduced, enabling modulation rates higher than the carrier relaxation rate. This speed enhancement originates from the regeneration characteristics of the nonlinear transfer function of the Fano resonance. Considering backward operation using the same device, we found the performance to be seriously degraded, i.e. $20 \mathrm{Gbit} / \mathrm{s}$ modulation cannot be achieved under the same conditions, proving that the advantage of the asymmetric structure originates from the larger coupling from the waveguide into the nanocavity.
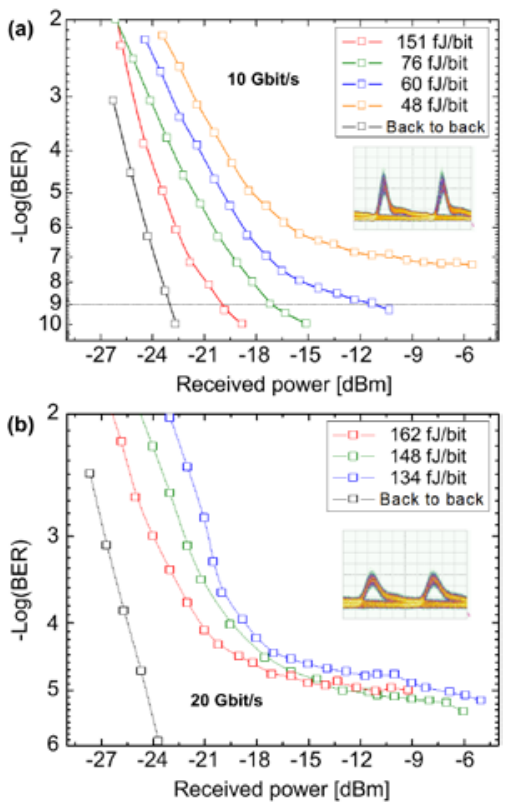

Fig. 5. BER measurements of modulated signal versus received power for different pump energies (colored markers) and back-toback signal (black markers) at (a) 10 and (b) 20 Gbit/s. Insets: Corresponding eye diagrams of the modulated signal.

For comparison, we also characterized another Fano structure where by shifting the $\mathrm{BH}$ by 2 lattice constants toward port 1 one can achieve a similar Fano line shape with $\gamma_{1} \gamma_{2} \approx 1$. Noting that we cannot compare it with a structurally symmetric Fano structure, where the PTE is centered right at the middle of the waveguide, because such a structure gives a Fano resonance with its transmission minimum red shift relative to its maximum, i.e., the Fano resonance has red parity [8], which is not suitable for optical switching based on carrier effects. This structure gives the lowest BER of the order of $10^{-3}$ at $20 \mathrm{Gbit} / \mathrm{s}$ operation, thus demonstrating the advantage of Fano structures employing broken symmetry.

\section{CONCLUSION}

We suggest and demonstrate a simple InP PhC asymmetric Fano switch based on an ultrasmall HO nanocavity. This structure has two distinctive features. First, a Fano resonance is achieved, exhibiting a large on-off contrast between the Fano resonance extrema, which enables large switching contrast, low pump-signal cross-talk and switching at speeds beyond the carrier relaxation rate dictated by the slow carrier recombination. Second, rather than the symmetric configurations usually considered, the structural mirror symmetry is broken, leading to a large coupling coefficient between the input port and cavity, which efficiently lowers the pump energy. These two elements allow the simultaneous achievements of error-free 10 Gbit/s RZ-OOK all-optical modulation with a pump energy as low as $60 \mathrm{fJ} / \mathrm{bit}$. For 20 Gbit/s operation, a BER three orders lower than the FEC limit is obtained. In both cases, long, telecom-grade, PRBS patterns of length $2^{31}-1$ were employed. The advantage relies entirely on optical design improvement and further performance improvements for ultrafast, low-energy on-chip signalprocessing for high-density integrated photonic circuits can be expected.

\section{ACKNOWLEDGMENT}

The authors acknowledge financial support from Villum Fonden via the NATEC (NAnophotonics for TErabit Communications) Centre.

\section{REFERENCES}

[1] D. A. B. Miller, Proc. IEEE 97, 1166 (2009)

[2] D. M. Beggs, T. P. White, L. O'Faolain, and T. F. Krauss, Opt. Lett., 33, 147, 149 (2008)

[3] K. Nozaki, T. Tanabe, A. Shinya, S. Matsuo, T. Sato, H. Taniyama, and M. Notomi, Nature Photon. 4, 477 (2010).

[4] C. Husko, A. De Rossi, S. Combrié, Q. Vy Tran, F. Raineri, and C. W. Wong, Appl. Phys. Lett. 94, 021111 (2009).

[5] A. Bazin, K. Lenglé, M. Gay, P. Monnier, L. Bramerie, R. Braive, G. Beaudoin, I. Sagnes, R. Raj, and F. Raineri. Appl. Phys. Lett. 104 011102 (2014).

[6] Fano, U. Phys. Rev. 124, 1866 (1961)

[7] K. Nozaki, A. Shinya, S. Matsuo, T. Sato, E. Kuramochi, and M. Notomi, Opt. Express, 21(10), 11877-11888 (2013).

[8] Y. Yu, M. Heuck, H. Hu, W. Q. Xue, C. Peucheret, Y. H. Chen, L. K. Oxenløwe, K. Yvind, and J. Mørk, Appl. Phys. Lett. 105, 061117 (2014).

[9] M. Heuck, S. Combrie, G. Lehoucq, S. Malaguti, G. Bellanca, S. Trillo, P. T. Kristensen, J. Mørk, J. P. Reithmaier, and A. De Rossi, Appl. Phys. Lett. 103, 181120 (2013).

[10] Y. Yu, E. Palushani, M. Heuck, S. Ek, N. Kuznetsova, P. Colman, P. T. Kristensen, C. Peucheret, L. K. Oxenløwe, S. Combrié, A. De Rossi, K. Yvind, and J. Mørk, Opt. Express 21, 31047-31061 (2013).

[11] D. Vukovic, Y. Yu, M. Heuck, S. Ek, N. Kuznetsova, P. Colman, E. Palushani, J. Xu, K. Yvind, L. K. Oxenløwe, J. Mørk, and C. Peucheret, IEEE Photon. Technol. Lett. 26, 257 (2013).

[12] Y. Yu, E. Palushani, M. Heuck, D. Vukovic, C. Peucheret, K. Yvind, and J. Mørk, Appl. Phys. Lett. 105, 071112 (2014)

[13] S. Combrié, G. Lehoucq, A. Junay, S. Malaguti, G. Bellanca, S. Trillo, L. Ménager, J. P. Reithmaier, and A. De Rossi, Appl. Phys. Lett. 103, 193510 (2013).

[14] T. Tanabe, K. Nishiguchi, A. Shinya, E. Kuramochi, H. Inokawa, M. Notomi, K. Yamada, T. Tsuchizawa, T.Watanabe, H. Fukuda, H. Shinojima, and S. Itabashi, Appl. Phys. Lett. 90, 031115 (2007).

[15] Q. V. Tran, S. Combrié, P. Colman, and A. De Rossi. Appl. Phys. Lett. 95, 061105 (2009)

[16] Y. Yu, M. Heuck, S. Ek, N. Kuznetsova, K. Yvind, and J. Mørk, Appl. Phys. Lett. 101, 251113 (2012).

[17] Y. Yu, H. Hu, L. K. Oxenløwe, K. Yvind, and J. Mørk, Opt. Lett. 40, 2357 (2015).

[18] M. Heuck, P. T. Kristensen, Y. Elesin, and J. Mørk. Opt. Lett. 38, 2466 (2013).

[19] Y. Yu, Y. H. Chen, H. Hu, W. Q. Xue, K. Yvind, and J. Mørk, Laser Photon. Rev. 9, 241-247 (2015)

[20] S. H. Fan, W. Suh, and J. D. Joannopoulos. J. Opt. Soc. Am. A 20, 569 (2003). 
